\title{
Effect of biopolymers on the characteristics and cytocompatibility of biocomposite nanofibrous scaffolds
}

\author{
Young Eun Kim and Young-Jin Kim
}

Polymer blending is one of the most effective methods for providing new and desirable biocomposites for biomedical applications. To develop a new potential scaffold, biocomposite nanofibers composed of poly(3-hydroxybutyrate-co-3hydroxyvalerate) (PHBV), gelatin and laminarin were prepared using an electrospinning process. The resulting nanofibers exhibited a fully interconnected pore structure and their average diameter varied according to the mixing ratio of polymers. According to the X-ray diffraction results, the crystalline phase of PHBV was hardly affected by the addition of biopolymers. However, the crystallinity obtained from the differential scanning calorimetry data increased with a decrease of gelatin content from 30.9 to $53.3 \%$. The water contact angles were markedly reduced by the addition of hydrophilic biopolymers from $94^{\circ}$ to $45^{\circ}$. The results from cytocompatibility tests showed that cell proliferation on the nanofibrous scaffolds increased with increasing gelatin content, whereas the improvement of proinflammatory cytokine expression was not proportional to the gelatin content. Therefore, PHBV/gelatin/laminarin (PHGL) biocomposite nanofibrous scaffolds appear to be promising biomaterials suitable for tissue engineering. Polymer Journal (2013) 45, 845-853; doi:10.1038/pj.2012.234; published online 30 January 2013

Keywords: biocomposite; biopolymer; nanofibrous scaffold; tissue engineering

\section{INTRODUCTION}

Tissue engineering has provided a new medical therapy as an alternative to conventional transplantation methods using polymeric biomaterials with or without living precursor cells. The fundamental approach in tissue engineering involves the fabrication of polymeric scaffolds with cells to produce a three-dimensional functional tissue suitable for implantation. ${ }^{1}$ Ideally, a scaffolding candidate should mimic the structure and biological function of native extracellular matrix (ECM), which have a pivotal role in controlling cell behavior. ${ }^{2}$ In addition, the scaffolding must support and define the threedimensional organization of the tissue-engineered space and maintain the normal state of differentiation within the cellular compartment. ${ }^{3}$

Principally, the scaffolds should be designed by mimicking the structure and biological function of native ECM, which provides mechanical support and regulates cell activities. Non-woven membrane composed of electrospun nanofibers is architecturally similar to the collagen structure of ECM, in which collagen multifibrils at a nanometer scale are composed of a three-dimensional network structure together with proteoglycans. ${ }^{4}$ Therefore, biomimetic matrices can be fabricated by electrospinning, which serve as a suitable environment for cell attachment and proliferation. ${ }^{2,5}$ In native tissues, the structural ECM proteins $(50-500 \mathrm{~nm}$ diameter fibers) are one to two orders smaller than the cell itself. This allows the cell to be in direct contact with many ECM fibers, thereby defining its three-dimensional orientation.

Polymers can be used as scaffolds to promote cell adhesion, maintenance of differentiated cells and the growth of cells, and help in the function of ECM. However, cell affinity towards synthetic polymers is generally poor as a consequence of their low hydrophilicity and lack of surface cell-recognition sites. ${ }^{6}$ In particular, acidic degradation products of synthetic biodegradable polymers-for instance, glycolic acid-induce inflammation at a wound site and thus biomedical applications of synthetic polymers can be limited. ${ }^{7}$ Improving the hydrophilicity, reducing the inflammatory response and incorporating the cell-recognition domains by using natural biopolymers such as ECM bioactive proteins can be carried out to enhance cell-scaffold interactions. ${ }^{8}$ Polymer blending is one of the most effective methods for providing new and desirable biocomposites for particular applications. ${ }^{9}$ Blending synthetic and natural biopolymers improves cell adhesion, and the degradation rate of the blended polymeric system can be modified depending on its applications. ${ }^{6,10}$

Poly(3-hydroxybutyrate-co-3-hydroxyvalerate) (PHBV) is a biodegradable polyester produced by various microorganisms as an 
intracellular reserve of carbon or energy. This biodegradable polymer has received much attention as a suitable material for various applications in medicine due to its attractive properties such as its natural origin, biocompatibility, non-toxicity, piezoelectricity and thermoplasticity. ${ }^{11,12}$ In addition, no acute inflammation, abscess formation or tissue necrosis has been observed in tissues adjacent to the implanted materials. However, it is a hydrophobic polyester without functional groups available for covalent immobilizing of cell-recognition signal molecules, which has limited its in vivo applications.

Gelatin is a natural biopolymer that can be obtained by thermal denaturation or physical and chemical degradation of collagen. It is commonly used in tissue engineering, and has been clinically proven as a temporary defect filler and wound dressing because of its biodegradability and biocompatibility. ${ }^{13,14}$ Gelatin is non-immunogenic compared with its precursor and can promote cell adhesion, migration, differentiation and proliferation. ${ }^{15,16}$ Moreover, owing to the large number of functional side groups, gelatin readily undergoes chemical crosslinking, which is very important for its use as a biomaterial.

Polysaccharides, including the D-glucopyranose unit linked by $\beta-1,3$ bonds, are known as essential constituents of the cell wall in fungi, and also as major storage carbohydrates in brown seaweed. ${ }^{17}$ It has been reported that $\beta-1,3$ glucan polymers have a wide variety of biological roles in plant and animal systems such as antitumor response, upregulation of NK cell toxicity and cytokine synthesis, anti-bacterial and anti-viral activity, and stimulation of hematopoiesis and wound healing. ${ }^{18,19}$ Among the $\beta-1,3$ glucan polymers, laminarin is the major storage polysaccharide of algae, and consists of glucose monomers that are joined together mainly by $\beta$-1,3-glycosidic bonds with some $\beta$-1,6-glycosidic bonds. Laminarin also possesses antimicrobial and antitumor activities by enhancing the host immune function. ${ }^{20}$

Electrospun polymers, in the form of a nanofibrous structure, have recently gained much interest for tissue engineering. ${ }^{2,4,5}$ The structural characteristics of nanofibrous membranes are necessary to enhance cell attachment and to expedite tissue ingrowth both in vitro and in vivo. In this study, the fabrication of PHBV/gelatin/laminarin (PHGL) biocomposite nanofibrous scaffolds was carried out by electrospinning. The influence of the mixing ratio of three components on the morphology of the resulting nanofibrous scaffolds was systematically examined. The effects of the biopolymers gelatin and laminarin on the surface hydrophilic property of the scaffolds were investigated. Furthermore, the cytocompatibility of the biocomposite scaffolds was evaluated by assaying cell proliferation and proinflammatory cytokine expression.

\section{EXPERIMENTAL PROCEDURE}

\section{Materials}

PHBV containing $5 \mathrm{wt} \%$ of hydroxyvalerate, teleostean gelatin from cold water fish skin (type A), laminarin from Laminaria digitata, 3-(4,5-dimethylthiazol2-yl)-2,5-diphenyltetrazoliumbromide (MTT), lipopolysaccharide (LPS), 2,2,2trifluoroethanol, formic acid and dimethyl sulfoxide were purchased from Sigma-Aldrich Co. (St Louis, MO, USA) and used without further purification. Dulbecco's modified Eagle's medium, fetal bovine serum and penicillinstreptomycin were obtained from Gibco BRL (Grand Island, NY, USA). Other reagents and solvents were commercially available and were used as received.

\section{Electrospinning}

The electrospinning setup utilized in this study consisted of a syringe and needle $(\mathrm{ID}=0.41 \mathrm{~mm})$, a ground electrode and a high voltage supply
(Chungpa EMT Co., Seoul, South Korea). The needle was connected to the high voltage supply, which could generate positive DC voltages up to $40 \mathrm{kV}$. For the electrospinning of PHGL nanofibers, a mixture of PHBV, gelatin and laminarin was first dissolved in a mixed solvent of 2,2,2-trifluoroethanol/ formic acid at a ratio of $8: 2$ to obtain a concentration of $8 \mathrm{wv} \%^{-1}$ solution. The weight ratio of PHBV, gelatin and laminarin in the mixed solutions was either 7:3:0, 7:2:1, 7:1:2 or 7:0:3. The PHGL solution was held in a $10 \mathrm{ml}$ syringe and was delivered into a needle spinneret by a syringe pump (KDS 100; KD Scientific Inc., Holliston MA, USA) with a mass flow rate of $1.5 \mathrm{ml} \mathrm{h}^{-1}$. The steel needle was connected to an electrode of high voltage supply and a grounded stainless-steel plate was placed at $15 \mathrm{~cm}$ distance from the needle tip to collect the nanofibers. The positive voltage applied to the polymer solutions was $15 \mathrm{kV}$. All experiments were carried out at room temperature and below $60 \%$ relative humidity. After electrospinning, the nanofibrous scaffold was carefully peeled off from the stainless-steel plate and put into an oven at $40{ }^{\circ} \mathrm{C}$ for $12 \mathrm{~h}$.

\section{Characterization of nanofibrous scaffolds}

The morphology of electrospun PHGL nanofibrous scaffolds was observed by a field emission-scanning electronic microscope (JSM-6335F; JEOL, Tokyo, Japan). Before SEM observation, all of the samples were coated with gold. The average diameter of nanofibers was determined by analyzing the SEM images with image analyzing software (Image-Pro Plus; Media Cybernetics Inc., Rockville, MD, USA). Attenuated total reflectance-Fourier transform infrared spectra of the samples were obtained using an ALPHA spectrometer (Bruker Optics, Billerica, MA, USA). The surface chemical compositions of the samples were analyzed by X-ray photoelectron spectroscopy (XPS) measurements (Quantera SXM, ULVAC-PHI Inc., Kanagawa, Japan). The incident $\mathrm{X}$-ray was monochromated $\mathrm{Al} \mathrm{K} \alpha$ radiation $(1486 \mathrm{eV})$. Survey scan and highresolution $\mathrm{C} 1 \mathrm{~s}$ peaks were recorded at a take-off angle of $45^{\circ}$ relative to the sample surface. X-ray diffraction measurements were carried out to characterize the crystalline phase of nanofibrous membranes with a PANalytical X-ray diffractometer X'Pert Pro using $\mathrm{Cu} \mathrm{K} \alpha$ radiation at $40 \mathrm{kV} / 30 \mathrm{~mA}$. The diffractograms were scanned in a $2 \theta$ range of $10-60^{\circ}$ at a rate of $2^{\circ} \mathrm{min}^{-1}$.

The thermal properties of nanofibrous scaffolds were evaluated using differential scanning calorimetry (Q2000; TA Instruments, New Castle, DE, USA) at a heating rate of $10{ }^{\circ} \mathrm{C} \mathrm{min}^{-1}$ under a nitrogen atmosphere from 30 to $300^{\circ} \mathrm{C}$. From the differential scanning calorimetry data, the crystallinity of the samples was calculated according to the following formula: ${ }^{21}$

$$
\text { Crystallinity }(\%)=\frac{\Delta H_{\mathrm{m}}^{\text {Sample }}}{f_{\mathrm{p}} \cdot \Delta H_{\mathrm{m}}^{*}} \times 100
$$

where $\Delta H_{\mathrm{m}}^{*}=146 \mathrm{Jg}^{-1}$ is the melting enthalpy for $100 \%$ crystalline PHBV and $f_{\mathrm{p}}$ is the PHBV weight fraction in the sample.

The contact angle between the water and the external surface of the nanofibrous membrane was measured to evaluate the membrane hydrophobicity using a contact angle meter (DSA 100; KRÜSS, Hamburg, Germany). To minimize experimental error, the contact angles were measured five times for each sample and then averaged.

\section{Cell viability assay}

In vitro cytotoxicity of PHGL biocomposite nanofibrous scaffolds was evaluated by a cell viability assay using mouse embryonic fibroblast cell line (NIH/3T3). All cultures were made in Dulbecco's modified Eagle's medium with $10 \%$ fetal bovine serum and were antibiotic/antimycotic in a humidified atmosphere under $5 \% \mathrm{CO}_{2}$ at $37^{\circ} \mathrm{C}$. When the cells reached $80 \%$ confluence, they were harvested using $0.25 \%$ trypsin-ethylenediamminetetraacetate acid and seeded into a new tissue culture plate for subculture.

The cell viability of the PHGL biocomposite nanofibrous scaffolds was investigated using the MTT assay. Before cell seeding, nanofibrous scaffolds were sterilized under ultraviolet light for $3 \mathrm{~h}$, followed by rinsing with phosphate-buffered saline and a culture medium five times, respectively. Then, mouse embryonic fibroblasts $\left(2 \times 10^{4}\right.$ cells per well $)$ were seeded onto the nanofibrous scaffolds in a 24-well tissue culture plate and cultured for 1, 3, 5 and 7 days at $37^{\circ} \mathrm{C}$. To evaluate the cell viability, $0.2 \mathrm{ml}$ of MTT solution $\left(5 \mathrm{mg} \mathrm{ml}^{-1}\right.$ in phosphate-buffered saline) was added to the cultured cells, 


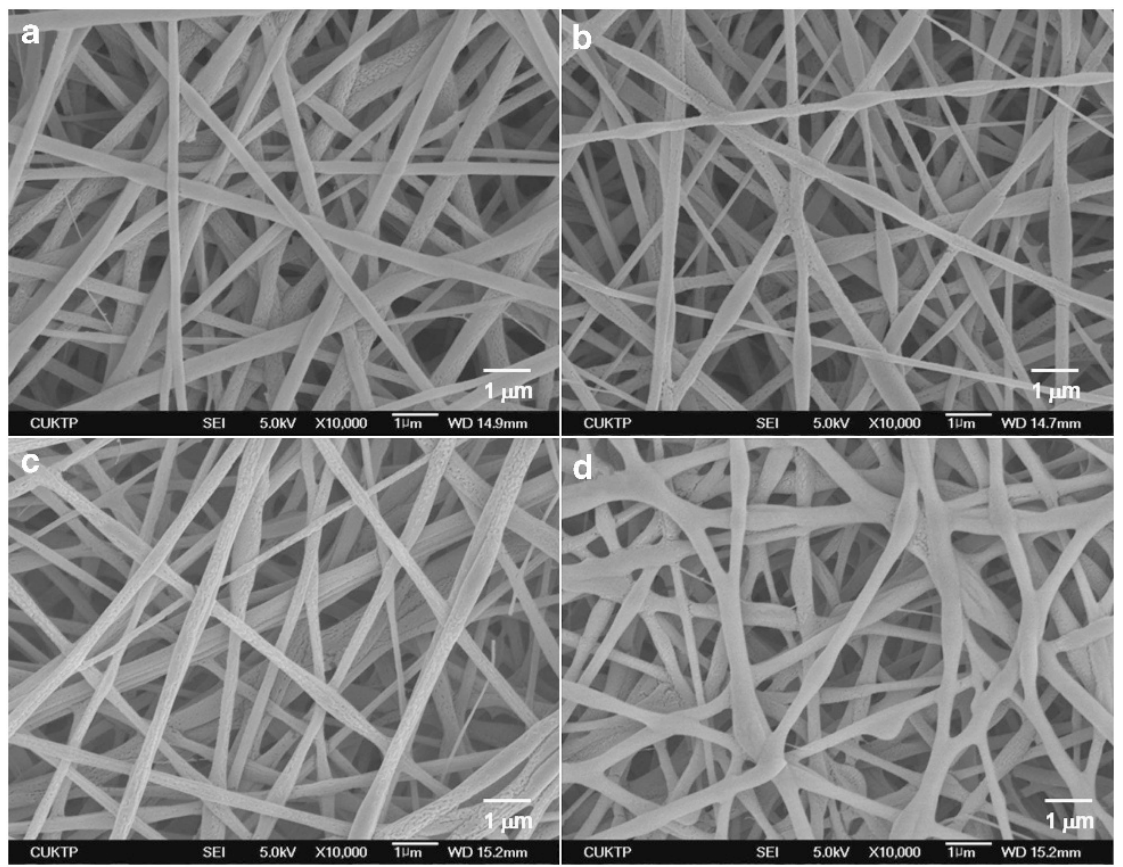

Figure 1 Scanning electronic microscope (SEM) micrographs of electrospun (a) poly(3-hydroxybutyrate-co-3-hydroxyvalerate) (PHBV)/gelatin/laminarin (PHGL)730, (b) PHGL721, (c) PHGL712 and (d) PHGL703 nanofibrous scaffolds.

which were further incubated for $4 \mathrm{~h}$ at $37^{\circ} \mathrm{C}$. After removing the remaining medium, $1 \mathrm{ml}$ of dimethyl sulfoxide was added to each well to solubilize the precipitate. Thereafter, $0.2 \mathrm{ml}$ of the resulting supernatant was transferred to a 96-well microplate and the optical density, which is proportional to the number of viable cells, was measured using a microplate reader (OPSYS-MR; Dynex Technology Inc., Chantilly, VA, USA). The mechanism of this assay is that metabolically active cells react with tetrazolium salt in the MTT reagent to produce water-insoluble formazan dye that can be observed at $570 \mathrm{~nm}$.

\section{Measurement of cytokine production}

The effect of biopolymers (gelatin and laiminarin) on the amount released of the proinflammatory factor interleukin-6 (IL-6) was determined with the murine macrophage cell line RAW 264.7, which was obtained from the Korean cell line bank. RAW 264.7 cells were cultured in Dulbecco's modified Eagle's medium supplemented with $10 \%$ heat-inactivated fetal bovine serum and were antibiotic/antimycotic in a humidified atmosphere under $5 \% \mathrm{CO}_{2}$ at $37^{\circ} \mathrm{C}$. For cytokine immunoassay, the cells $\left(1 \times 10^{5}\right.$ cells per well $)$ were seeded onto the nanofibrous scaffolds in a 24-well tissue culture plate and pre-cultured for $24 \mathrm{~h}$ at $37^{\circ} \mathrm{C}$. After that, $1 \mu \mathrm{g} \mathrm{ml}{ }^{-1}$ of LPS was added to the cell culture plate to activate cells for $24 \mathrm{~h}$. The concentration of IL- 6 was determined using a commercial mouse enzyme-linked immunosorbent assay kit (R\&D Systems, Minneapolis, MN, USA). The kit was used according to the manufacture's specifications.

\section{RESULTS AND DISCUSSION}

\section{Morphology of electrospun nanofibrous scaffolds}

The use of scaffolding materials for tissue engineering is an attempt to mimic the ECM in connective tissues. Electrospun nanofibrous scaffolds could be an ideal structural matrix that resembles the fibrous structure of collagen bundles in the ECM. Furthermore, ultrafine nanofiber structures are thought to enhance cell adhesion, migration and proliferation. , $^{2,5}$ In this study, biocomposite nanofibers were prepared by electrospinning from PHBV/gelatin/ laminarin blend solutions with weight ratios between PHBV, gelatin and laminarin of 7:3:0 (PHGL730), 7:2:1 (PHGL721), 7:1:2 (PHGL712) and 7:0:3 (PHGL703), respectively, at a concentration of $8 \mathrm{wv} \%^{-1}$.

Figure 1 shows SEM micrographs of electrospun PHGL biocomposite nanofibrous scaffolds. The resulting electrospun nanofibrous scaffolds exhibited a fully interconnected pore structure with narrow pore size distribution. The average diameter of nanofibers reduced with increasing the concentration of laminarin in the solution, which was $360 \pm 35 \mathrm{~nm}$ for PHGL730, $313 \pm 47 \mathrm{~nm}$ for PHGL721, $287 \pm 31$ for PHGL712 and $283 \pm 25 \mathrm{~nm}$ for PHGL703. This result may be related to a change of solution viscosity through the use of laminarin, which has relatively low viscosity compared with gelatin. The solution viscosity decreased from 376 to $250 \mathrm{cP}$ with increasing the content of laminarin. It has previously been reported that the average diameter of PHBV nanofibers decreases through the addition of a lowmolecular-weight collagen peptide, caused by a reduction of the solution viscosity. ${ }^{22}$ This result suggests that the solution viscosity is the most important parameter in electrospinning, and can affect the morphology of nanofibers, with the result that higher viscosity gives rise to an increase in fiber diameter.

\section{Properties of nanofibrous scaffolds}

Attenuated total reflectance-Fourier transform infrared analysis was carried out to allow the surface characterization of PHGL nanofibers in the range of $400-4000 \mathrm{~cm}^{-1}$. PHGL730 exhibited a characteristic absorption band at $3300 \mathrm{~cm}^{-1}$ associated with an $\mathrm{N}-\mathrm{H}$ stretching vibration in gelatin (Figure 2a). According to a previous report, a free $\mathrm{N}-\mathrm{H}$ stretching vibration occurs in the range from 3400 to $3440 \mathrm{~cm}^{-1}$, and when the $\mathrm{N}-\mathrm{H}$ group of peptides is involved in hydrogen bonding, the position is shifted to lower frequencies, usually around $3300 \mathrm{~cm}^{-1} .{ }^{23}$ From this result, it seems that many of the N-H groups of gelatin are involved in intermolecular hydrogen bonding. In our experiments, the characteristic absorption band was shifted from 


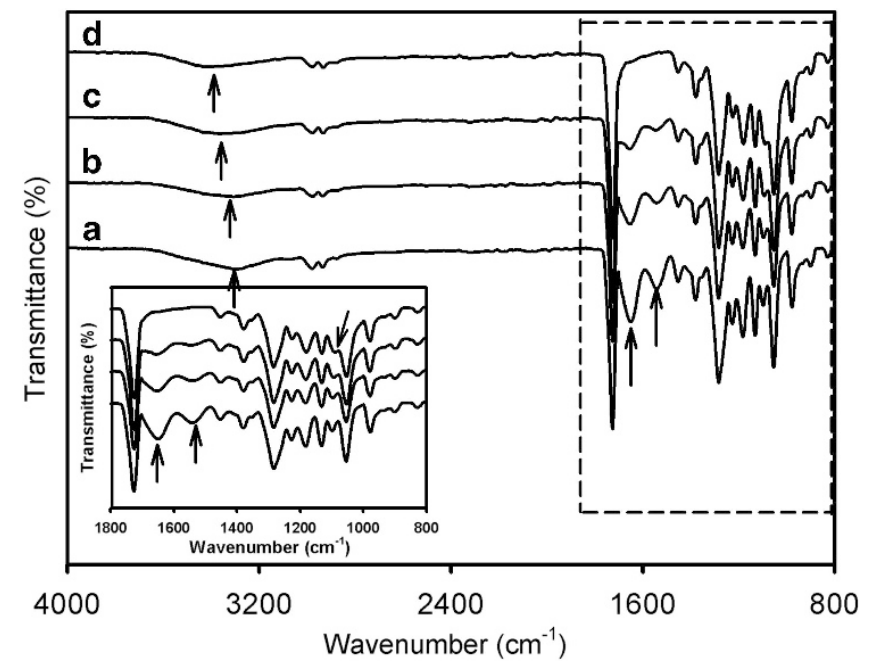

Figure 2 Attenuated total reflectance-Fourier transform infrared (ATR-FTIR) spectra of (a) poly(3-hydroxybutyrate-co-3-hydroxyvalerate) (PHBV)/gelatin/ laminarin (PHGL)730, (b) PHGL721, (c) PHGL712 and (d) PHGL703 nanofibrous scaffolds.

3300 to $3390 \mathrm{~cm}^{-1}$ with decreasing gelatin content due to the reduction of intermolecular hydrogen bonding as shown in Figure 2. Moreover, common bands of gelatin appeared at approximately $1644 \mathrm{~cm}^{-1}$ (amide I) and $1540 \mathrm{~cm}^{-1}$ (amide II) in PHGL730 nanofiber, corresponding to the stretching vibration of the $\mathrm{C}=\mathrm{O}$ bond, and the coupling of $\mathrm{N}-\mathrm{H}$ bond bending and $\mathrm{C}-\mathrm{N}$ bond stretching, respectively. ${ }^{24}$ These two characteristic peaks diminished with reducing gelatin content, disappearing in the PHGL703 nanofiber, but an additional characteristic shoulder peak was observed at $1080 \mathrm{~cm}^{-1}$ and ascribed to the typical $\beta$-glycosidic bonds of laminarin. ${ }^{25,26}$ In addition, a characteristic peak centered at $1727 \mathrm{~cm}^{-1}$ was also observed in all samples, which was derived from the $\mathrm{C}=\mathrm{O}$ stretching of ester groups present in the molecular chain of highly ordered crystalline PHBV. ${ }^{27}$

The surface chemical composition of the biocomposite nanofibrous scaffolds was determined by XPS. Figure 3 shows survey scan spectra of PHGL nanofibers. As can be seen from Figure 3, PHGL nanofibers containing gelatin (PHGL730, PHGL721 and PHGL712 nanofibers) exhibited the same XPS spectra patterns. These spectra consisted of three separated peaks assigned to PHBV, gelatin and laminarin, which corresponded to C 1s $(285 \mathrm{eV}), \mathrm{N}$ 1s $(400 \mathrm{eV})$ and $\mathrm{O} 1 \mathrm{~s}(532 \mathrm{eV})$. However, in the case of PHBV and PHGL703 nanofibers, only two peaks, C 1 s $(285 \mathrm{eV})$ and $\mathrm{O} 1 \mathrm{~s}(532 \mathrm{eV})$, assigned to PHBV and laminarin, were observed owing to the absence of gelatin.

Moreover, to obtain further insight into the chemical bonds present on the surface of the nanofibers, high-resolution XPS C 1s spectra can be deconvoluted using a curve fitting technique. Figure 4 shows the decomposed $\mathrm{C} 1 \mathrm{~s}$ peaks of nanofibers and the fractions of the different carbon functional groups are given in Table 1. As shown in Figure 4, the spectra from the nanofibers were resolved into four components: a peak at $284.3 \mathrm{eV}$ corresponding to a $\mathrm{C}-\mathrm{C}$ bond, a peak at $285.4 \mathrm{eV}$ assigned to a $\mathrm{C}-\mathrm{O}$ bond, a peak at $286.3 \mathrm{eV}$ ascribed to a $\mathrm{C}-\mathrm{N}$ bond and a peak at $288.2 \mathrm{eV}$ due to a $\mathrm{C}=\mathrm{O}$ bond. ${ }^{28}$ The area of the $\mathrm{C} 1 \mathrm{~s}$ peak of the $\mathrm{C}-\mathrm{O}$ bond increased from 24.1 to $41.0 \%$ with an incremental increase in laminarin content, which can be attributed to an increase in the ether moiety on the surface of PHGL nanofibers through the addition of laminarin (Table 1). However, the C 1s peak

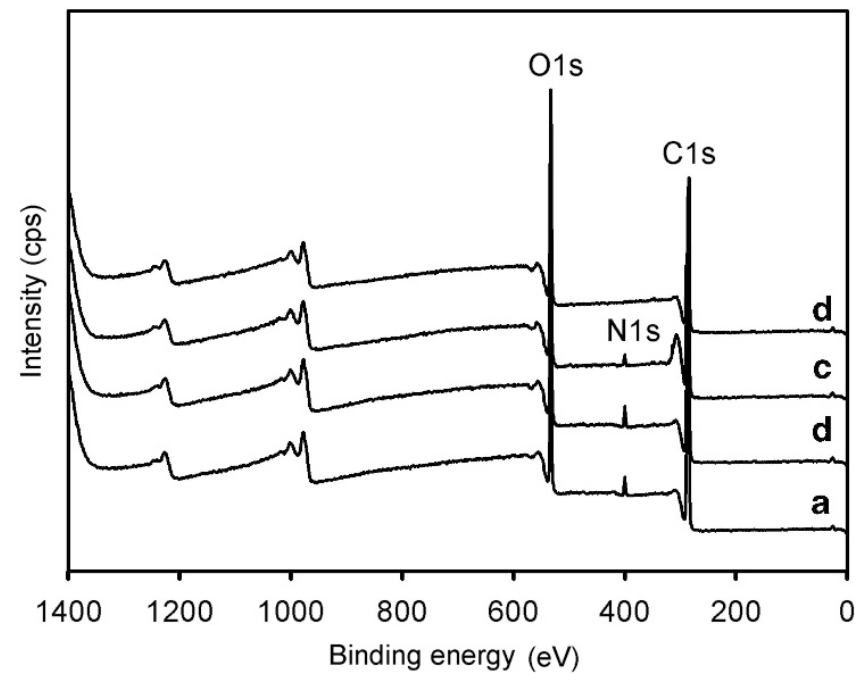

Figure 3 X-ray photoelectron spectroscopy (XPS) survey scan spectra (a) poly(3-hydroxybutyrate-co-3-hydroxyvalerate) (PHBV)/gelatin/laminarin (PHGL) 730, (b) PHGL721, (c) PHGL712 and (d) PHGL703 nanofibrous scaffolds.

ascribed to the $\mathrm{C}-\mathrm{N}$ functional group of the peptide bond diminished from 12.5 to $5.6 \%$ with reducing the gelatin content and then vanished from the PHGL703 nanofiber on account of the absence of gelatin as described above.

Crystallographic analysis was performed using X-ray diffraction to elucidate the change in crystal structure of the PHGL nanofibers. It has been reported that PHBV crystallizes in poly(3-hydroxybutyrate) (PHB) crystalline lattice at a 3-hydroxyvalerate (HV) content that is lower than $50 \mathrm{~mol} \%$. However, PHBV having over $50 \mathrm{~mol} \%$ of HV content exhibites a poly(3-hydroxyvalerate) (PHV) crystalline lattice. ${ }^{29}$ PHBV used in this study had a 5 wt $\%$ HV content; therefore, it crystallized in the PHB crystalline lattice and had the same crystal structure as that of PHB with the orthorhombic unit cell. All of the samples exhibited two strong diffraction peaks at around $13.6^{\circ}$ and $17.0^{\circ}$, which corresponded to the (020) and (110) planes, respectively (Figure 5). In addition, three other weak diffraction peaks were observed at $19.5^{\circ}, 25.3^{\circ}$ and $27.1^{\circ}$ and ascribed to the (0 21 1), (1 21 ) and (0 4 0) planes. However, the gelatin and laminarin content hardly affected the diffraction peaks arising from PHBV as shown in Figure 5. This means that gelatin and laminarin recrystallization did not occur during the electrospinning.

To obtain quantitative data regarding the degree of crystallinity of nanofibers, differential scanning calorimetry measurements were carried out. As shown in Figure 6 and Table 2, the PHGL nanofibers showed almost the same melting temperature, which ranged from 167.8.0 to $168.5^{\circ} \mathrm{C}$. The gelatin and laminarin content barely influenced the melting temperature of the polymer membranes, although there was a considerable change in the melting enthalpy. The crystallinity values calculated from the melting enthalpy of nanofibers increased from 30.9 to $53.3 \%$ with reducing the gelatin content in nanofibers. This arises because of the amorphous character of gelatin. ${ }^{30}$

To improve the affinity between the scaffold and the cells, it is important to observe the physical state on the surface of the materials that are in contact with the cells because the cell attachment and proliferation depend on the hydrophilicity, charge on the material's surface, existence of a connecting sequence and surface texture. Among them, hydrophilicity is an important characteristic that would 

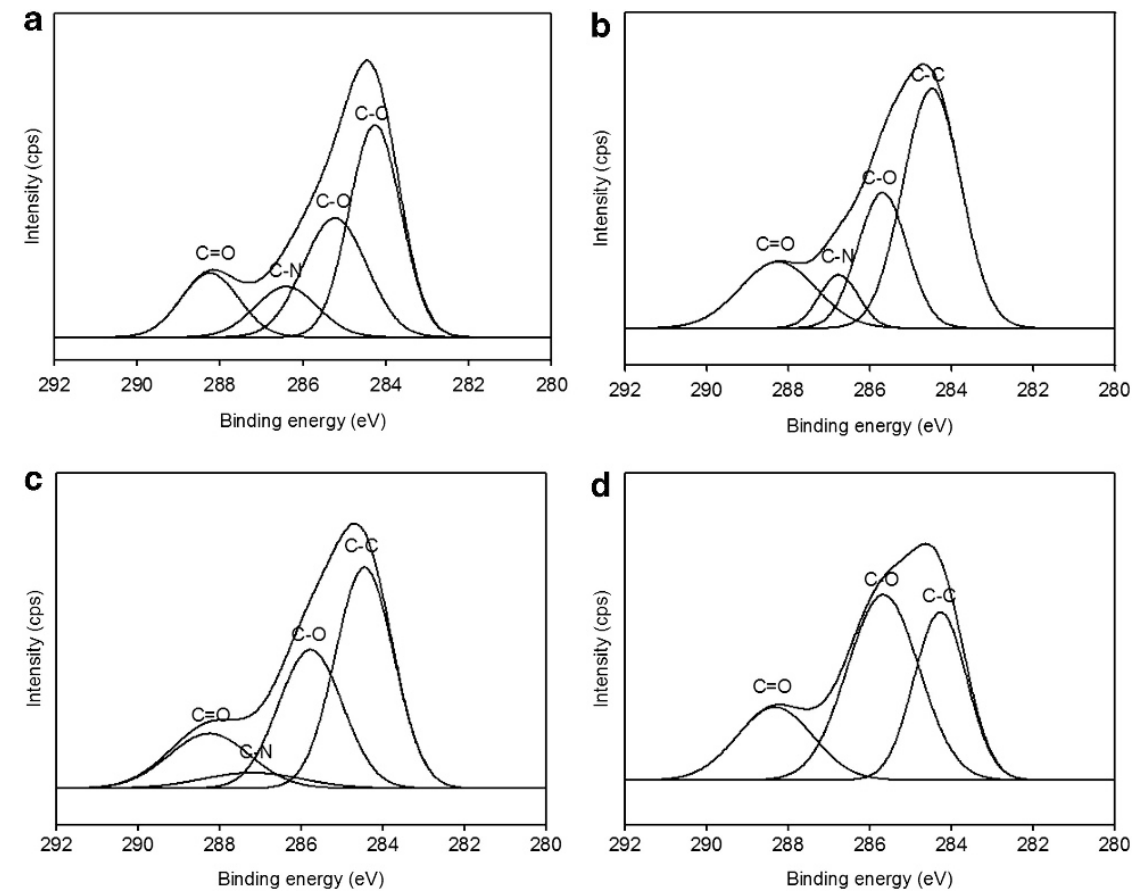

Figure 4 X-ray photoelectron spectroscopy (XPS) C 1s spectra with peak deconvolutions for (a) poly(3-hydroxybutyrate-co-3-hydroxyvalerate) (PHBV)/gelatin/ laminarin (PHGL)730, (b) PHGL721, (c) PHGL712 and (d) PHGL703 nanofibrous scaffolds.

Table 1 Fraction of carbon functional groups from high-resolution $\mathrm{C}$ 1s XPS peaks

\begin{tabular}{lcccc}
\hline & $\begin{array}{c}C-C(\%) \\
(284.3 \mathrm{eV})\end{array}$ & $\begin{array}{c}C-O \\
(285.4 \mathrm{eV})\end{array}$ & $\begin{array}{c}C-N(\%) \\
(286.3 \mathrm{eV})\end{array}$ & $\begin{array}{c}C=O(\%) \\
(288.2 \mathrm{eV})\end{array}$ \\
\hline PHGL730 & 43.0 & 24.1 & 12.5 & 20.4 \\
PHGL721 & 50.0 & 29.7 & 7.6 & 18.3 \\
PHGL712 & 46.3 & 32.0 & 5.6 & 16.1 \\
PHGL703 & 38.9 & 41.0 & - & 20.1 \\
\hline
\end{tabular}

Abbreviations: PHBV, poly(3-hydroxybutyrate-co-3-hydroxyvalerate); PHGL, PHBV/gelatin/ laminarin; XPS, X-ray photoelectron spectroscopy.

affect the performance of a biomaterial in application. A more hydrophilic surface is favorable to cell attachment and spreading. ${ }^{31}$ The water contact angle has been used as an indication of hydrophilicity in many studies. ${ }^{32,33}$ The contact angle data from the nanofibrous scaffolds are shown in Figure 7. PHBV nanofibers showed hydrophobic properties, in which the water contact angle of the PHBV nanofibers was $\sim 94^{\circ}$ (data not shown). The contact angles were reduced by the addition of hydrophilic biopolymers and, peculiarly, the incremental increase in gelatin content caused a marked decrease in the contact angles, which were $45^{\circ}$ for PHGL730, $61^{\circ}$ for PHGL721, $78^{\circ}$ for PHGL712 and $87^{\circ}$ for PHGL703. These results suggest that gelatin was more effective in diminishing the contact angle of nanofibers than laminarin because of the relatively high hydrophilicity of gelatin.

\section{Cell viability on nanofibrous scaffolds}

Tissue engineering scaffolding must promote cell growth and physiological function and should maintain normal states of cell differentiation. Thus, the cytocompatibility of PHGL biocomposite

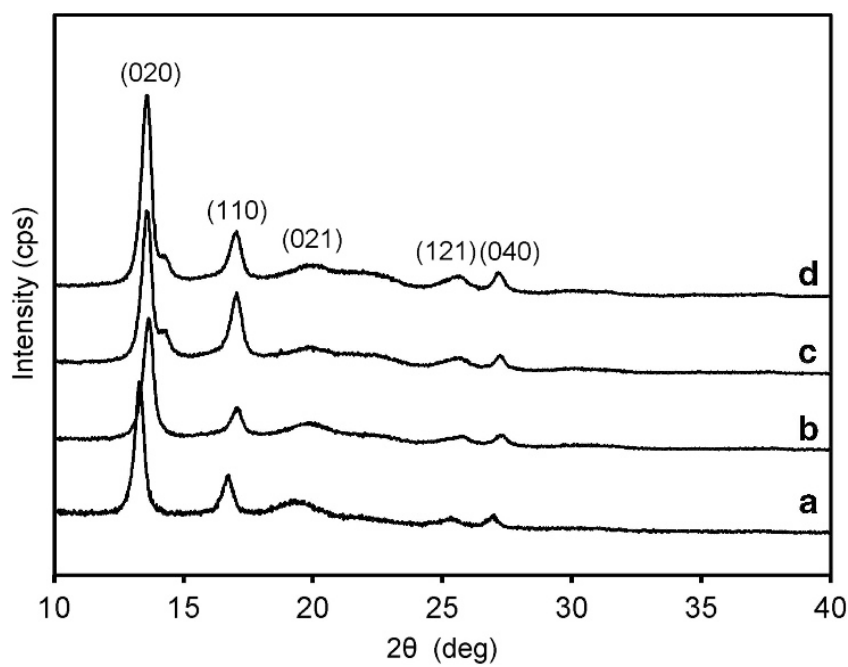

Figure 5 X-ray diffraction patterns of (a) poly(3-hydroxybutyrate-co-3hydroxyvalerate) (PHBV)/gelatin/laminarin (PHGL)730, (b) PHGL721, (c) PHGL712 and (d) PHGL703 nanofibrous scaffolds.

nanofibrous scaffolds was examined as a potential candidate for scaffolds to be used in tissue engineering. An MTT assay was carried out to evaluate the cell viability of mouse embryonic fibroblasts (NIH/3T3) on PHGL nanofibrous scaffolds. NIH/3T3 proliferated faster on PHGL nanofibrous scaffolds compared with PHBV nanofibers during the incubation period (Figure 8). The number of attached cells in the early stage was similar in all samples. However, cell proliferation on the nanofibrous scaffolds increased with increased gelatin content, indicating that gelatin effectively accelerates the proliferation and differentiation of NIH/3T3 cells. Among the 


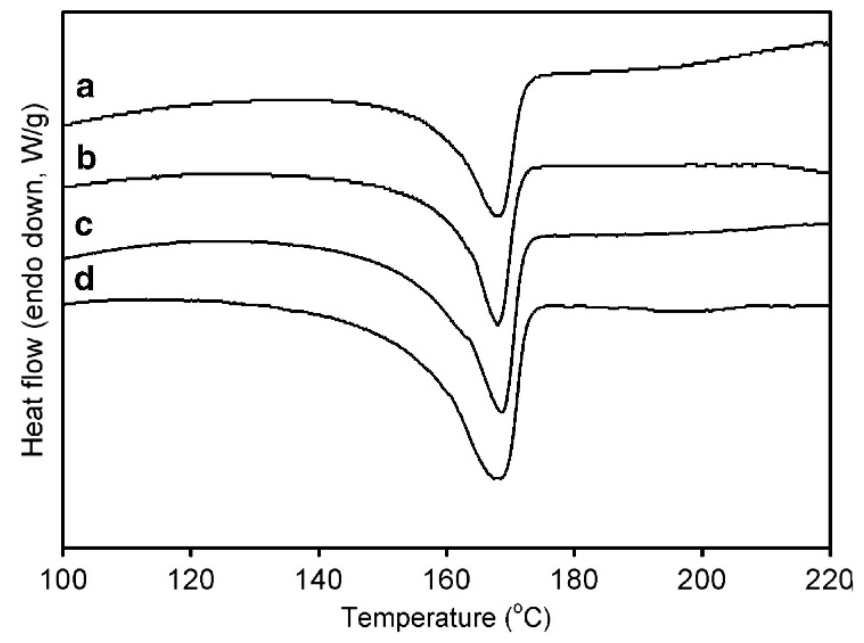

Figure 6 Differential scanning calorimetry (DSC) curves of (a) poly(3hydroxybutyrate-co-3-hydroxyvalerate) (PHBV)/gelatin/laminarin (PHGL)730, (b) PHGL721, (c) PHGL712 and (d) PHGL703 nanofibrous scaffolds.

Table 2 Thermal properties and crystallinity of PHGL nanofibrous scaffolds

\begin{tabular}{lccc}
\hline & Melting temperature $\left({ }^{\circ} \mathrm{C}\right)$ & Melting enthalpy $\left(\mathrm{Jg}^{-1}\right)$ & Crystallinity (\%) \\
\hline PHGL730 & 168.0 & 31.6 & 30.9 \\
PHGL721 & 168.0 & 41.3 & 40.4 \\
PHGL712 & 168.5 & 46.3 & 45.3 \\
PHGL703 & 167.8 & 54.5 & 53.3 \\
\hline
\end{tabular}

Abbreviations: PHBV, poly(3-hydroxybutyrate-co-3-hydroxyvalerate); PHGL, PHBV/gelatin/laminarin. nanofibers, cell proliferation was significantly higher on PHGL730 nanofibers in comparison with other nanofibers. It is well known that gelatin is a biodegradable, biocompatible and non-immunogenic material, which is suitable for biomedical applications. In addition, gelatin contains acidic residues, such as lysine and arginine, that have a positive charge. Cells tend to adhere much more strongly to substrates with acidic groups than to substrates with basic or neutral groups, because cells generally have negative charges on their surfaces. Therefore, gelatin can promote cell adhesion, migration, differentiation and proliferation. ${ }^{15,16}$ Laminarin also stimulated the proliferation of NIH/3T3 cells by improving the hydrophilicity of the scaffolds as shown in Figure 8. According to a previous report, laminarin exhibits excellent antioxidative activities, which can positively influence cell viability. ${ }^{34}$ However, the effect of laminarin on cell growth is lower than that of gelatin.

Cell morphology and the interaction between cells and nanofibers were also observed during the incubation period. SEM micrographs showed that NIH/3T3 adhered and spread on the surface of PHGL nanofibrous scaffolds (Figure 9). In addition, cell proliferation increased time dependently in all samples. Among them, cells were covering the entire PHGL730 nanofibrous scaffold and formed a cell monolayer after 7 days of culture. This may be evidence of the ability of gelatin to stimulate the growth of NIH/3T3. In addition, NIH/3T3 cells also spread over most of the surfaces of the PHGL721 and PHGL712 nanofibers after 7 days in culture. These results are consistent with a previous report that showed that fibroblasts proliferate well on porous scaffolds that are composed of gelatin and $\beta$-glucan. ${ }^{35}$ However, the growth of NIH/3T3 was decelerated relative to a decline in the gelatin content as shown in the results from the MTT assay.

\section{Expression of proinflammatory cytokines}

IL-6 is known to have a crucial role in mediating the inflammatory and immune responses that are initiated by infection or injury.

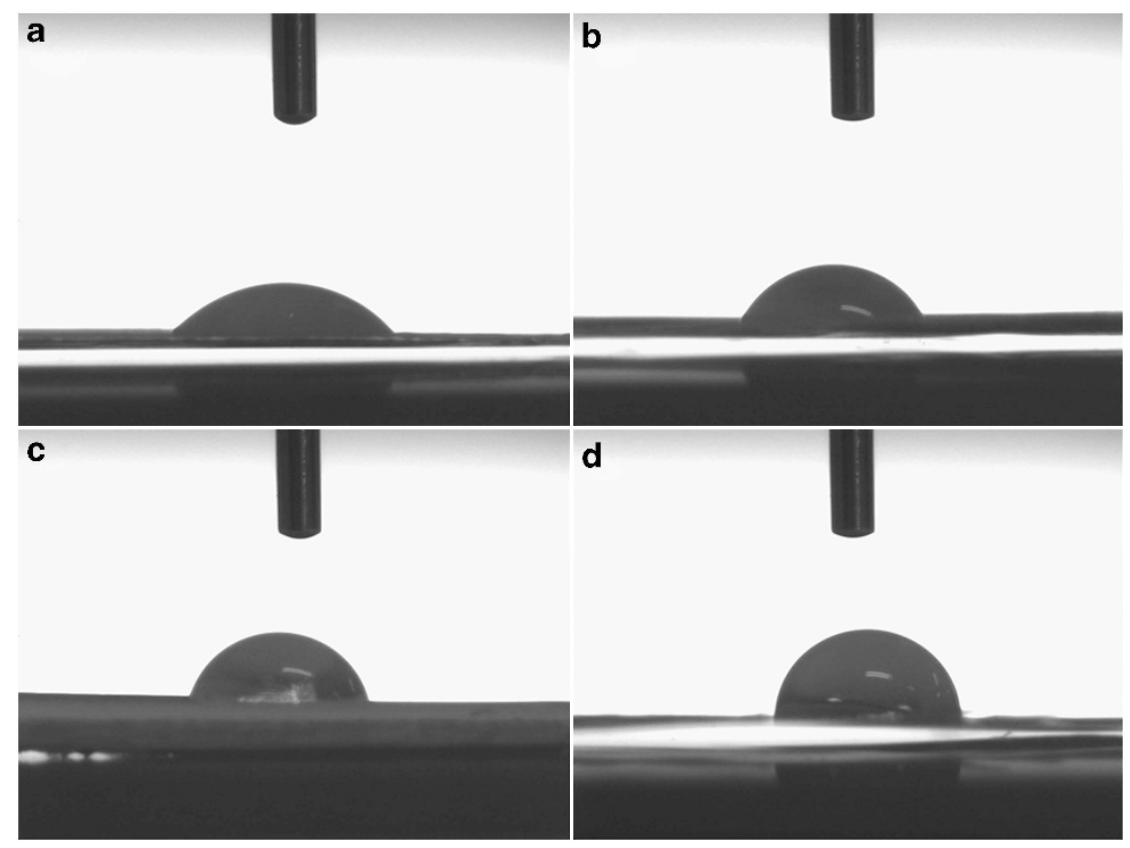

Figure 7 Contact angle changes of (a) poly(3-hydroxybutyrate-co-3-hydroxyvalerate) (PHBV)/gelatin/laminarin (PHGL)730, (b) PHGL721, (c) PHGL712 and (d) PHGL703 nanofibrous scaffolds. 
Elevated IL-6 levels are associated with severe foreign-body reactions to implanted materials. ${ }^{36}$ In addition, significant release of cytokines can deteriorate the cell viability and induce histological damage. ${ }^{37}$ Excessive expression of IL-6 can be observed in the early days after transplantation of artificial substitutes for various human tissues. Thus, the expression of IL- 6 in cell cultures was analyzed to assess the cytocompatibility of PHGL nanofibrous scaffolds. PHBV nanofibers

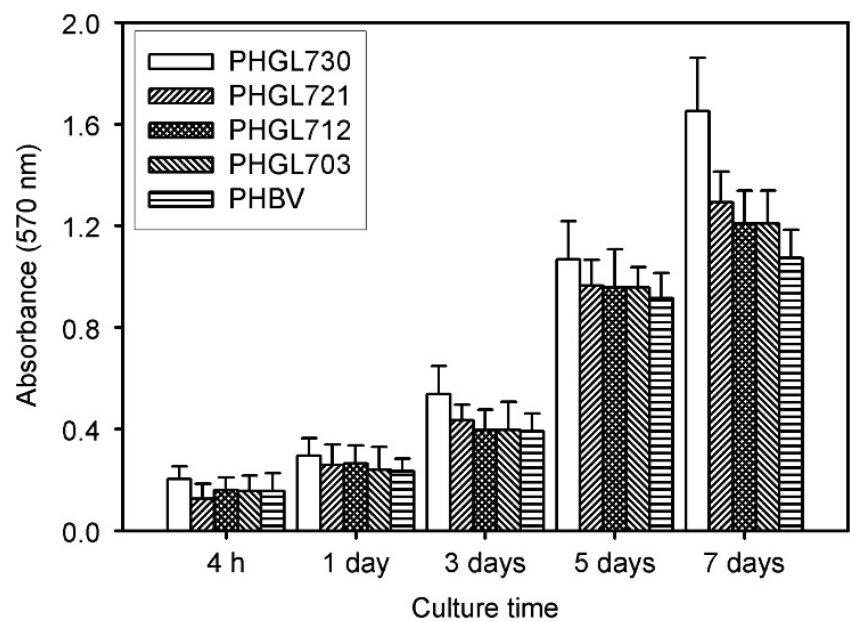

Figure 8 Cell viability of NIH/3T3 cells incubated onto poly(3hydroxybutyrate-co-3-hydroxyvalerate) (PHBV) and PHBV/gelatin/laminarin (PHGL) nanofibrous scaffolds as a function of culture time. stimulated production of the proinflammatory cytokine IL-6 considerably, as shown in Figure 10. IL-6 expression by the PHBV nanofibers was very high and nearly the same as IL-6 expression on the control tissue culture plate (data not shown). It was previously found that PHBV can induce macrophage activation in RAW 264.7 cells and that this activation requires the cells to be in direct contact with this material. ${ }^{38}$ This means that PHBV can be contaminated

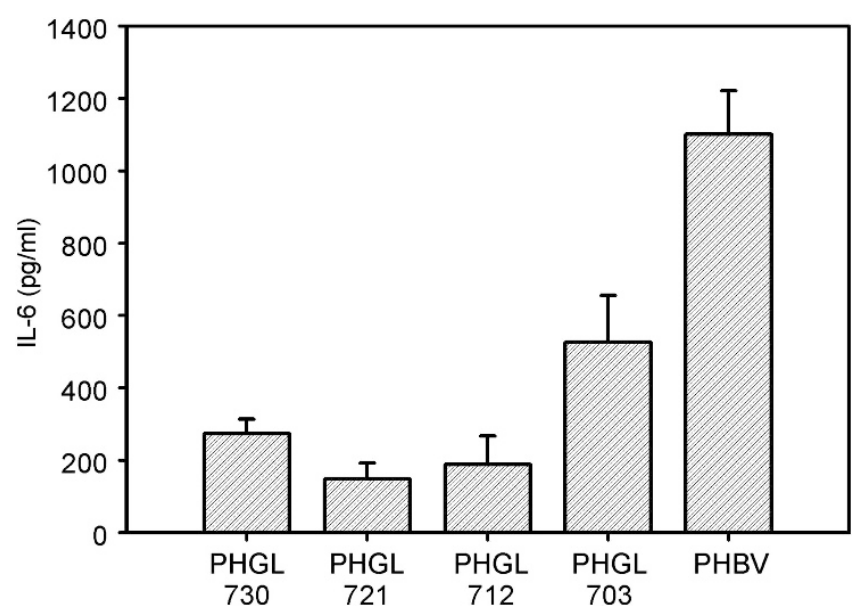

Figure 10 Levels of interleukin (IL)-6 released from RAW 264.7 cells after $24 \mathrm{~h}$ incubation onto poly(3-hydroxybutyrate-co-3-hydroxyvalerate) (PHBV) and PHBV/gelatin/laminarin (PHGL) nanofibrous scaffolds.

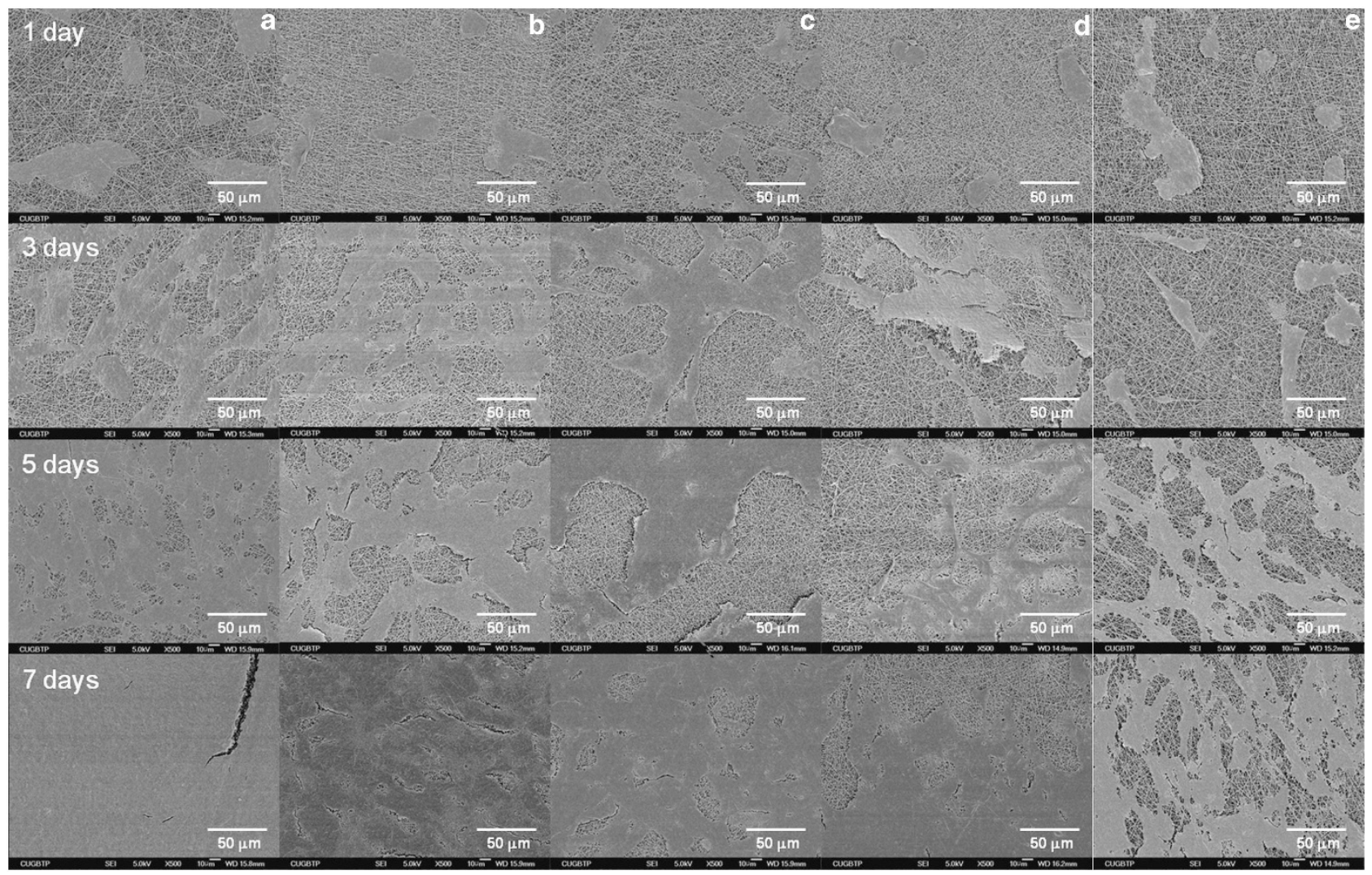

Figure 9 Scanning electronic microscope (SEM) micrographs of NIH/3T3 cells grown on (a) poly(3-hydroxybutyrate-co-3-hydroxyvalerate) (PHBV)/gelatin/ laminarin (PHGL)730, (b) PHGL721, (c) PHGL712, (d) PHGL703 and (e) PHBV nanofibrous scaffolds for different periods of time (1, 3, 5 and 7 days). 
easily by LPS triggering macrophage activation. This endotoxin, LPS, is a major factor contributing to the poor biocompatibility seen in polyhydroxyalkanoate-based medical devices. Moreover, polyhydroxyalkanoate samples contain bacterially derived impurities such as long-chain fatty acids of $\beta$-hydroxypalmitic acid and saturated $\beta$-hydroxytetradecanoic acid. ${ }^{39}$ These impurities can also induce the proinflammatory response to PHBV.

The addition of biopolymers substantially reduced the proinflammatory response to PHBV. When compared with PHBV nanofibers, all of PHGL nanofibrous scaffolds showed remarkably reduced levels of IL-6 expression. In particular, the nanofibrous scaffolds that included both gelatin and laminarin, the PHGL721 and PHGL712 nanofibers, exhibited very low amounts of IL-6 secretion, meaning that nanofibers that include gelatin/laminarin may cause a lesser cellular inflammatory reaction and have more acceptable cytocompatibility. This is because gelatin does not express antigenicity in physiological conditions and is completely resorbable in vivo. ${ }^{40}$ Furthermore, laminarin can modulate the metabolic and toxic response to LPS administration by changing the pattern of immune cells present in the liver tissue, resulting in the restraint of cytokine production by monocytes via LPS stimulation. ${ }^{41}$ In addition, it is of interest to note that hydrophilic surfaces show decreased expression of proinflammatory cytokines. ${ }^{36}$ Therefore, controlling the mixing ratio between gelatin and laminarin is the most important factor for reducing IL-6 expression.

\section{CONCLUSION}

One of the key factors in tissue engineering is the creation of threedimensional scaffolds that have a structural and functional resemblance to native ECM and a suitable degradation rate. Porous scaffolds for tissue engineering should have high porosity to allow the accommodation of a large number of cells, as well as fully interconnected pores to facilitate the uniform distribution of cells and the diffusion of oxygen and nutrients. To date several synthetic materials have been used in attempts to meet these criteria, including polymers of lactic acid and glycolic acid, polycaprolactone and their copolymers. These materials have many favorable properties, although unfortunately they have not performed to expectations in the clinical setting because of their low hydrophilicity, lack of surface cellrecognition sites and induction of the inflammatory response. In this study, PHGL biocomposite nanofibers produced through the electrospinning process were introduced for use as a scaffold for the growth of fibroblasts. The resulting nanofibers showed a porous morphology formed by interlaying of the fibers. The characteristics and cytocompatibility of nanofibrous scaffolds were significantly influenced by the introduction of the biopolymers gelatin and laminarin. The contact angles of these scaffolds were significantly reduced by the addition of hydrophilic biopolymers, particularly gelatin. Moreover, cell attachment and proliferation improved as the ratio of gelatin increased in the mixture, whereas increases in proinflammatory cytokine expression were not proportional to the gelatin content. Therefore, controlling the mixing ratio of the biopolymers gelatin and laminarin is the most important factor for improving the cytocompatibility of the synthetic biodegradable polymer PHBV. Consequently, PHGL biocomposite nanofibrous scaffolds will be useful for tissue engineering applications.

\section{ACKNOWLEDGEMENTS}

This research was supported by a Marine Biomaterials Research Center grant from the Marine Biotechnology Program, which is funded by the Ministry of Land, Transport and Maritime Affairs, Korea.
1 Rho, K. S., Jeong, L., Lee, G., Seo, B. M., Park, Y. J., Hong, S. D., Roh, S., Cho, J. J., Park, W. H. \& Min, B. M. Electrospinning of collagen nanofibers: effects on the behavior of normal human keratinocytes and early-stage wound healing. Biomaterials 27, 1452-1461 (2006)

2 Ghasemi-Mobarakeh, L., Prabhakaran, M. P., Morshed, M., Nasr-Esfahani, M. H. \& Ramakrishna, S. Electrospun poly( $\varepsilon$-caprolactone)/gelatin nanofibrous scaffolds for nerve tissue engineering. Biomaterials 29, 4532-4539 (2008).

3 Matthews, J. A., Wnek, G. E., Simpson, D. G. \& Bowlin, G. L. Electrospinning of collagen nanofibers. Biomacromolecules 3, 232-238 (2002).

4 Nishida, T., Yasumoto, K., Otori, T. \& Desaki, J. The network structure of corneal fibroblasts in the rat as revealed by scanning electron microscopy. IOVS 29 , 1887-1890 (1988).

5 Smith, L. A. \& Ma, P. X. Nano-fibrous scaffolds for tissue engineering. Colloid Surf. B 39, 125-131 (2004).

6 Ciardelli, G., Chiono, V., Vozzi, G., Pracella, M., Ahluwalia, A., Barbani, N., Cristallini, C. \& Giusti, P. Blends of poly-(E-caprolactone) and polysaccharides in tissue engineering applications. Biomacromolecules 6, 1961-1976 (2005).

7 Nair, L. S. \& Laurencin, C. T. Biodegradable polymers as biomaterials. Prog. Polym Sci. 32, 762-798 (2007).

8 Koh, H. S., Yong, T., Chan, C. K. \& Ramakrishna, S. Enhancement of neurite outgrowth using nano-structured scaffolds coupled with laminin. Biomaterials 29, 3574-3582 (2008)

9 Cheng, M., Deng, J., Yang, F., Gong, Y., Zhao, N. \& Zhang, X. Study on physical properties and nerve cell affinity of composite films from chitosan and gelatin solutions. Biomaterials 24, 2871-2880 (2003).

10 Sabir, M. I., Xu, X. \& Li, L. A review on biodegradable polymeric materials for bone tissue engineering applications. J. Mater. Sci. Mater. Med. 44, 5713-5724 (2009).

11 Lenz, R. W. \& Marchessault, R. H. Bacterial polyesters: biosynthesis, biodegradable, plastics and biotechology. Biomacromolecules 6, 1-8 (2005).

12 Ke, Y., Wang, Y. J., Ren, L., Zhao, Q. C. \& Huang, W. Modified PHBV scaffolds by in situ UV polymerization: structural characteristic, mechanical properties and bone mesenchymal stem cell compatibility. Acta Biomater. 6, 1319-1336 (2010).

13 Chang, M. C., Ko, C. \& Douglas, W. H. Preparation of hydroxyapatite-gelatin nanocomposite. Biomaterials 24, 2853-2862 (2003).

14 Yung, C. W., Wu, L. Q., Tullman, J. A., Payne, G. F., Bentley, W. E. \& Barbari, T. A. Transglutaminase crosslinked gelatin as a tissue engineering scaffold. J. Biomed. Mater. Res. A 83, 1039-1046 (2007).

15 Burg, K. J., Porter, S. \& Kellam, J. F. Biomaterial developments for bone tissue engineering. Biomaterials 21, 2347-2459 (2000).

16 Cui, Y. L., Hou, X., Qi, A. D., Wang, X. H., Wang, H., Cai, K. Y., Yin, Y. J. \& Yao, K. D. Biomimetic surface modification of poly (L-lactic acid) with gelatin and its effects on articular chondrocytes in vitro. J. Biomed. Mater. Res. A 66, 770-778 (2003).

17 Miyanishi, N., Iwamoto, Y., Watanabe, E. \& Oda, T. Induction of TNF- $\alpha$ production from human peripheral blood monocytes with $\beta$-1,3-glucan oligomer prepared from laminarin with $\beta-1,3$-glucanase from bacillus clausii NM-1. J. Biosci. Bioeng. 95, 192-195 (2003)

18 Goldman, R. C. Biological response modification by $\beta$-D-glucans. Annu. Rep. Med. Chem. 30, 129-138 (1995).

19 Ross, G. D., Větvička, V., Yan, J., Xia, Y. \& Větvičková, J. Therapeutic intervention with complement and $\beta$-glucan in cancer. Immunopharmacology 42, 61-74 (1999).

20 Strinvastava, R. \& Kulshrestha, D. K. Bioactive polysaccharides from plants. Phytochemistry 28, 2877-2883 (1989).

21 Carli, L. N., Crespo, J. S. \& Mauler, R. S. PHBV nanocomposites based on organomodified montmorillonite and halloysite: the effect of clay type on the morphology and thermal and mechanical properties. Composites Part A 42, 1601-1608 (2011).

22 Kim, Y. J., Bae, H. I., Kwon, O. K. \& Choi, M. S. Three-dimensional gastric cancer cell culture using nanofiber scaffold for chemosensitivity test. Int. J. Biol. Macromol. 45 , 65-71 (2009).

23 Nagai, T., Suzuki, N. \& Nagashima, T. Collagen from common minke whale (Balaenoptera acutorostrata) unesu. Food Chem. 111, 296-301 (2008).

24 Muyonga, J. H., Cole, C. G. B. \& Duodu, K. G. Fourier transform infrared (FTIR) spectroscopic study of acid soluble collagen and gelatin from skins and bones of young and adult nile perch (Lates niloticus). Food Chem. 86, 325-332 (2004).

25 Methacanon, P., Weerawatsophon, U., Tanjak, P., Rachtawee, P. \& Prathumpai, W. Interleukin-8 stimulating activity of low molecular weight $\beta$-glucan depolymerized by $\gamma$-irradiation. Carbohydr. Polym. 86, 574-580 (2011).

26 Novák, M., Synytsya, A., Gedeon, O., Slepička, P., Procházka, V., Synytsya, A., Blahovec, J., Hejlová, A. \& Čopíková, J. Yeast $\beta(1-3),(1-6)$-D-glucan films: preparation and characterization of some structural and physical properties. Carbohydr. Polym. 87, 2496-2504 (2012).

27 Padermshoke, A., Kastumoto, Y., Sato, H., Ekgasit, S., Noda, I. \& Ozaki, Y. Surface melting and crystaliization behavior of polyhydroxyalkanoates studied by attenuated total reflection infrared spectroscopy. Polymer (Guildf) 45, 6547-6554 (2004)

28 Chen, J. P. \& Su, C. H. Surface modification of electrospun PLLA nanofibers by plasma treatment and cationized gelatin immobilization for cartilage tissue engineering. Acta Biomater. 7, 234-243 (2011)

29 Yoshie, N., Saito, M. \& Inoue, Y. Structural transition of lamella crystals in a isomorphous copolymer, poly(3-hydroxybutyrate-co-3-hydroxyvalerate). Macromolecules 34, 8953-8960 (2001). 
30 Bergo, P. \& Sobral, P. J. A. Effects of plasticizer on physical properties of pig skin gelatin films. Food Hydrocolloids 21, 1285-1289 (2007).

31 Wei, J. H., Yoshinari, M., Takemoto, S., Hattori, M., Kawada, E., Liu, B. L. \& Oda, Y. Adhesion of mouse fibroblasts on hexamethyldisiloxane surfaces with wide range of wettability. J. Biomed. Mater. Res. B 81B, 66-75 (2007).

32 Yeo, M. G., Lee, H. \& Kim, G. H. Polycaprolactone, $\beta$-tricalcium phosphate, and collagen nanofibers: fabrication, physical properties, and in vitro cell activity for bone tissue regeneration. Biomacromolecules 12, 502-510 (2011).

33 Wu, F., Liu, C., O'Neill, B., Wei, J. \& Ngothai, Y. Fabrication and properties of porous scaffold of magnesium phosphate/polycaprolactone biocomposite for bone tissue engineering. Appl. Surf. Sci. 258, 7589-7595 (2012).

34 Choi, J. I., Kim, H. J. \& Lee, J. W. Structural feature and antioxidant activity of low molecular weight laminarin degraded by gamma irradiation. Food Chem. 129, 520-523 (2011).

35 Lee, S. B., Jeon, H. W., Lee, Y. W., Lee, Y. M., Song, K. W., Park, M. H., Nam, Y. S. \& Ahn, H. C. Bio-artificial skin composed of gelatin and $(1 \rightarrow 3)$, $(1 \rightarrow 6)-\beta$-glucan. Biomaterials 24, 2503-2511 (2003).
36 Brodbeck, W. G., Voskerician, G., Ziats, N. P., Nakayama, Y., Matsuda, T. \& Anderson, J. M. In vivo leukocyte cytokine mRNA responses to biomaterials are dependent on surface chemistry. J. Biomed. Mater. Res. A 64, 320-329 (2003).

37 Bowers, W., Blaha, M., Alkhyyat, A., Sankovich, J., Kohl, J., Wong, G. \& Patterson, D. Artificial human skin: cytokine, prostaglandin, Hsp70 and histological responses to heat exposure. J. Dermatol. Sci. 20, 172-182 (1999).

38 Wu, A. C. K., Grødahl, L., Jack, K. S., Foo, M. X., Trau, M., Hume, D. A. \& Cassady, A. I. Reduction of the in vitro pro-inflammatory response by macrophages to poly(3-hydroxybutyrate-co-3-hydroxyvalerate). Biomaterials 27, 4715-4725 (2006).

39 Shishatskaya, E. I. \& Volova, T. G. A comparative investigation of biodegradable polyhydroxyalkanoate films as matrices for in vitro cell cultures. J. Mater. Sci. Mater. Med. 15, 915-923 (2004).

40 Bigi, A., Cojazzi, G., Panzavolta, S., Roveri, N. \& Rubini, K. Stabilization of gelatin films by crosslinking with genipin. Biomaterials 23, 4827-4832 (2002).

41 Neyrinck, A. M., Mouson, A. \& Delzenne, N. M. Dietary supplementation with laminarin, a fermentable marine $\beta(1-3)$ glucan, protects against hepatotoxicity induced by LPS in rat by modulating immune response in the hepatic tissue. Int. Immunopharmacol. 7, 1497-1506 (2007). 